\title{
Le déversoir à seuil épais
}

\section{Broadcrested weir}

\author{
PAR L.-J. TISON \\ Professeur a x'Univensité de Gand
}

English synopsis, p. 418.

1. On s'est longtemps contenté de la formule de Bélanger pour déterminer le débit sur ces déversoirs. Se basant sur l'hypothèse du débil maximum, Bélanger avait trouvé que :

$$
\mathrm{Q}=0,385 \cdot \sqrt{ } 2 g \cdot c^{3 / 2}
$$

Q étant le débit par mètre de largeur du déversoir à seuil horizontal, et

c. étant la hauteur d'eau à l'amont du déversoir par rapport au seuil.

Si la vitesse d'amenée n'est pas négligeable,

c doit être augmenté de $\frac{\mathrm{U}^{2}}{2 g}$, $\mathrm{U}$ étant la vitesse

moyenne à l'amont du déversoir. Le seuil est supposé suffisamment arrondi à l'amont pour que le coefficient de contraction puisse être pris égal à l'unité. Cette formule fut soumise à diverses vérifications, notamment par BAzIN (1), $\mathrm{et}$, dans les dernières années, le déversoir en question a fait l'objet d'assez nombreuses études parmi lesquelles nous signalerons celles d'Escande (2), de Hunter Rouse (3), de J. G. Woodbunn (4), laquelle donna lieu à une discussion très intéressante à laquelle prirent part H. W. King, Sherman, M. Woodward, Dayid L. Yarnell, Boris A. BakmmetefF, D. D. Curtis, E. Lindouist, R. L. Parshall.

2. L'écoulement sur un déversoir à seuil épais peut être considéré comme étant constitué par un écoulement dans un canal de longueur égale à celle du seuil, alimenté à l'amont par un réservoir avec production d'une inflexion de la surface supérieure et se déversant à l'aval en nappe libre soumise à la pression atmosphérique sur ses faces tant inférieure que supérieure. On peut cependant rencontrer les diverses formes habituelles de nappes autres que la nappe libre.

3. Etude de l'inflexion à l'amont. - Considérons un réservoir $A B$ (fig. 1), de forme quelconque : ce sera dans notre probleme le canal à l'amont du déversoir à seuil épais. Ce déversoir

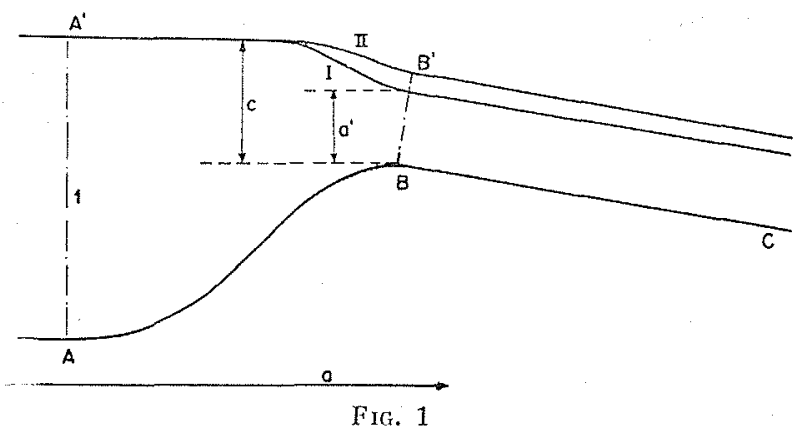

est figuré par la partie BC. La section $\mathrm{BB}^{\prime}$ dans laquelle les filets sont redevenus sensiblement parallèles, peut être considérée comme étant hydrostatique. Nous admettrons que l'action du frottement soit négligeable, de telle façon que les vitesses en $\mathrm{BB}^{\prime}$ puissent être considérées comme étant uniformément réparties. La forme du fond à l'amont immédiat de B permet d'admettre que la contraction est nulle.

Appliquons au liquide du réservoir, compris cntre la section $\mathrm{BB}^{\prime}$ normale au fond et une section $\mathrm{AA}^{\prime}$ verticale, par exemple, le théorème des quantités de mouvement projeté sur un axe horizontal $a$. En AA', les vitesses sont devenues 
très faibles et la section $\mathrm{AA}^{\prime}$ est évidemment hydrostatique.

L'équation en question peut s'écrire, en négligeant les vitesses en $\mathrm{AA}^{\prime}$ :

$o_{1} \frac{p_{1}}{\gamma}=\left(\omega \frac{p}{\gamma}+\frac{q \mathrm{U}}{g}\right) \sqrt{1-i^{2}-\frac{\Phi_{a}}{\gamma}}$

L'indice 1 se rapporte à la section $\mathrm{AA}^{\prime}$, tandis que les lettres sans indice sont relatives à la section $\mathrm{BB}^{\prime}$. Les $\omega$ sont les sections, les $p$ sont les pressions aux centres de gravité des sections, $\gamma$ est le poids spécifique de l'eau, tandis que $q$ est le débit et $U$ est la vitesse uniforme dans la section $\mathrm{BB}^{\prime} . \Phi_{a}$ représente la projection sur l'axe $a$ des réactions normales du fond du réservoir. Les réactions tangentielles de ce fond sont en effet négligeables, comme les vitesses dans le réservoir $A B$. $i$ vaut sin $\partial$, ótant l'angle de $B C$ avee l'horizontale.

Soit encore $a^{\prime}$ la hauteur d'eau verticale dans la section $\mathrm{BB}^{\prime}$. On suppose que la forme de la section w est telle que l'angle formé par la tangente it son contour avec l'horizontale croit avec $h$.

Imaginons maintenant que, par suite d'autres circonstances, linflexion, au lieu de donner une ligne I présente une ligne II située au-dessus de la première. La valeur de $a^{\prime}$ va varier avec l'inflexion et il en sera de même de $\Phi_{a}$.

Le remplacement par l'inflexion II de la situation primitivement considérée a pour effet d'augmenter les réactions normales du fond dans une région oil elles donnent des composantes négatives dans l'expression de $\Phi_{a}$. Il en résulte que $\Phi_{a}$ diminue quand $a^{\prime}$ augmente, de sorte que $\frac{d \Phi_{a}}{d a^{\prime}} \leqslant 0$.

D'autre part, on peut déduire de l'équation 1 , en remărquant que son premier membre n'est pas influencé par la variation de $a^{\prime}$ :

$\frac{1}{\gamma} \frac{d \Phi_{a}}{d a^{\prime}}=\frac{d}{d a^{\prime}}\left(\omega \frac{p}{\gamma}+\frac{\omega \mathrm{U}^{2}}{g}\right) \sqrt{ } 1-i^{2}$

$\frac{p}{\gamma}$ est précisément la distance verticale du centre de gravité de w au plan horizontal passant par $B^{\prime}$ et si $\mathrm{BB}^{\prime}$ vaut $h$, l'équation 2 peut s'écrire :

$$
\begin{gathered}
\frac{1}{\gamma} \cdot \frac{d \Phi_{a}}{d a^{\prime}}=\left[\frac{d\left(\omega \cdot \frac{p}{\gamma}\right)}{d h} \cdot \frac{d h}{d a^{\prime}}\right. \\
\left.+\frac{2 \omega \cdot \mathrm{U}}{g} \cdot \frac{d \mathrm{U}}{d a^{\prime}}+\frac{\mathrm{U}^{2}}{g} \cdot \frac{d \omega}{d h} \cdot \frac{d h}{d a^{\prime}}\right] \sqrt{ } 1-i^{2},
\end{gathered}
$$

et comme

$$
\frac{d\left(\omega \cdot \frac{p}{\gamma}\right)}{d h}=\omega \sqrt{ } 1-i^{2} \text { et } \mathrm{U}=\sqrt{ } 2 g\left(c-a^{\prime}\right) \text {, }
$$

$c$ étant la hauteur d'eau du réservoir avant l'inflexion par rapport à l'horizontale de $\mathrm{B}$, on a encore :

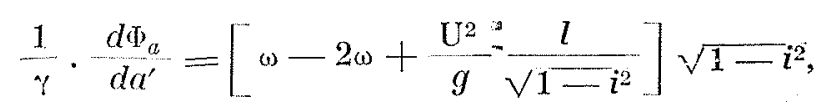

$l$ étant la largeur à la surface de la section $\omega$ : $\frac{d \omega}{d h}$ vaut en efret $l$.

On a done:

$-\frac{1}{\gamma} \frac{d \Phi_{a}}{d \alpha^{\prime}}=\omega\left(\sqrt{ } \mathbf{1}-i^{2}-\frac{q^{2}}{g} \cdot \frac{l}{\omega^{3}}\right)$

La condition $\frac{d \Phi_{a}}{d a^{\prime}} \leqslant 0$ conduit par conséquent à :

$$
\omega\left(\sqrt{1-i^{2}}-\frac{q^{2}}{g} \cdot \frac{l}{\omega^{3}}\right) \geqslant 0 .
$$

On ne modifie rien à l'écoulement étudié cidessus si on imagine qu'on amène l'arête inférieure d'une vanne au contact de la veine liquide : l'inflexion devient alors de l'affleurement. A chaque levée de la vanne fictive en simple contact avec la veine, correspond une valeur du débit. La dérivation de l'équation $q=\omega \sqrt{2} g\left(c-a^{\prime}\right)$ conduit à :

$$
\sqrt{1-i^{2}}=\frac{q^{2}}{g} \cdot \frac{l}{\omega^{3}}
$$

c'est-à-dire que le débit est maximum pour une valeur $a_{m}^{\prime}$ de $a^{\prime}$ igale a $\mathrm{H}_{1}^{\prime} \vee 1-i^{\prime}, \mathrm{H}_{1}^{\prime}$ étant la hauteur critique (hauteur caracléristique du ressaut) pour une valeur du débit égale au débit maximum. On doit en effet remarquer que $\mathrm{H}_{1}$, solution de l'équation $\sqrt{1-i^{2}}=\frac{q^{2}}{g} \cdot \frac{l}{\omega^{3}}$, varie avec la valeur du débit.

$\mathrm{Si}$ on recherche la variation de $\mathrm{H}_{1}$ avec $a^{\prime}$, on devra se reporter à l'équation suivante:

$$
\sqrt{1-\ddot{i}^{3}}=-\frac{q^{2}}{g} \cdot \frac{l_{1}}{\omega_{1}^{3}}
$$

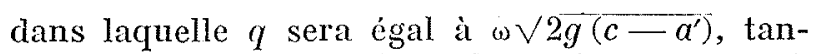
dis que $l_{1}$ et $\omega_{1}$ seront les valeurs de la largeur à la surface et de la section pour $h=\mathrm{H}_{1}$.

L'équation 4 peut donc s'écrire :

$$
\sqrt{1-i^{2}}=\left(\frac{\omega}{\omega_{1}}\right)^{2} \cdot 2 \cdot\left(c-a^{\prime}\right) \cdot \frac{l_{1}}{\omega_{1}} .
$$


Premier cas. - $a^{\prime}$ est inférieur à $a_{m^{\prime}}$. $-\mathrm{Si} a^{\prime}$ décroît, $c-a^{\prime}$ croît, et on sait que le débit décroit. Mais comme $\mathrm{H}_{1}$ est une fonction croissante du débit, $\mathrm{H}_{1}$ décroît dans le cas considéré. Par contre, $\frac{\omega_{1}}{l_{1}}$ etant une fonction croissante de $\mathrm{H}_{1}$, $\frac{l_{1}}{\omega_{1}}$ croît quand $\mathrm{H}_{1}$ décroît et quand par conséquent $a^{\prime}$ décroît. Le troisième facteur du second membre $\left(\frac{\omega}{\omega_{1}}\right)^{2}$ doit par conséquent décroître avec $a^{\prime}$ puisque les deux autres facteurs varient en sens opposé.

Or, pour $a^{\prime}=a_{m}{ }^{\prime}, \omega=\omega_{1}$ puisque $h \vee 1-i^{2}$ $=a^{\prime}=a_{m}{ }^{\prime}=\mathrm{H}_{1} \vee 1-i^{2}$. De sorte que si $a^{\prime}$ devient inférieur à $a_{m}{ }^{\prime}, \omega$ devient plus petit que $\omega_{1}$, c'est-à-dire que $h$ ou $\frac{a^{\prime}}{\sqrt{1-i^{\grave{2}}}}$ devient inférieur à $\mathrm{H}_{1}$.

Deuxième cas : $a^{\prime}$ est supérieur à $a_{m}{ }^{\prime}$. - On peut montrer d'une façon analogue que dans ce cas, $h$ ou $\frac{a^{\prime}}{\sqrt{1-i^{2}}}$ est supérieur à $\mathrm{H}_{1}$.

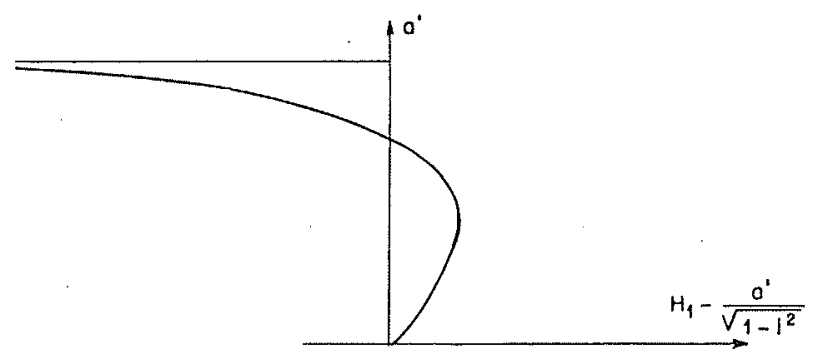

FIG. 2

Le diagramme ci-contre donne, par conséquent la variation de $\mathrm{H}_{1}--\frac{a^{\prime}}{\sqrt{1-i^{2}}}$ avec $a^{\prime}$.

Si nous nous reportons maintenant à la condition 4, w étant nécessairement positif, elle se ramène à :

$$
\sqrt{1-i^{2}}--\frac{q^{2}}{g} \cdot \frac{l}{\omega^{3}} \geqslant 0 .
$$

Or, on peut aisément montrer que

$$
\sqrt{1-i^{2}}-\frac{q^{2}}{g} \frac{l}{\omega^{3}}
$$

est une fonction constamment croissante de $h$ s'annulant pour $h=\mathrm{H}_{1}$ (diagramme 3 ).

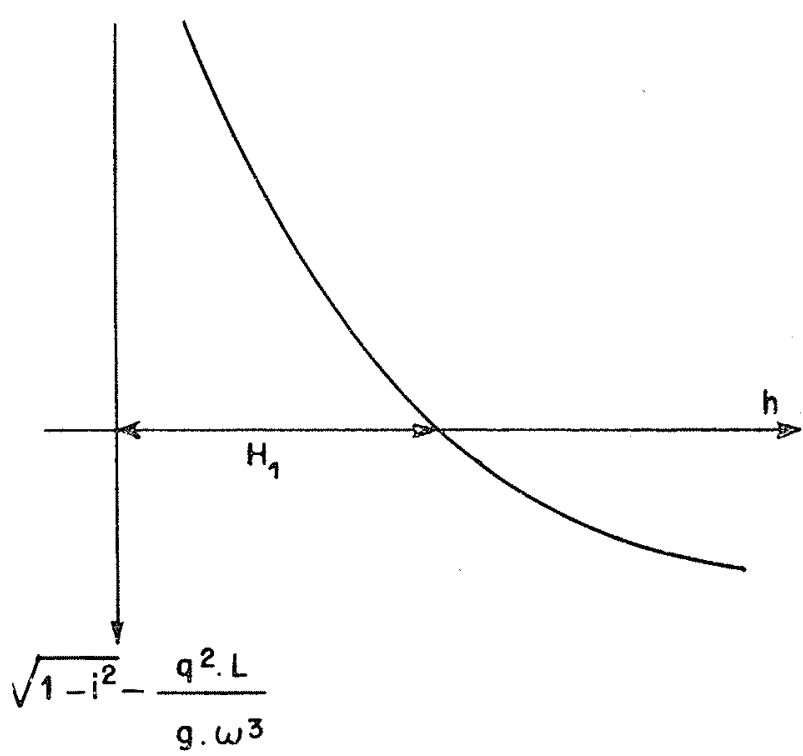

FIG. 3

On a vu ci-dessus que pour $a^{\prime}<a_{m}{ }^{\prime}, h$ est inférieur à $\mathrm{H}_{1}$ correspondant au débit déterminé par $a$. Le diagramme 3 montre que, dans ce cas, la fonction $\sqrt{1-i^{2}}-\frac{q^{2}}{g} \frac{l}{\omega^{3}}$ prend une valeur négative, de sorte qu'on ne peut satisfaire à lá condition 4.

Par contre, $h$ est supérieur à $\mathrm{H}_{1}$ pour $a^{\prime}>a_{m}{ }^{\prime}$, de sorte que la fonction $\sqrt{1-i^{2}}-\frac{q^{2}}{g} \frac{l}{\omega^{3}}$ est alors positive ce qui permet de satisfaire à la condition 4.

$$
\text { Enfin } h=\mathrm{H}_{1}{ }^{\prime} \text { si } a^{\prime}=a_{m^{\prime}} \text { et } \sqrt{1-i^{-3}}-\frac{q^{2} l}{g-\frac{l}{\omega^{3}}}
$$

est nul : la condition 4 est donc aussi salisfaite dans ce cas.

De tout ceci résulte que linflexion conduit toujours à une première hauteur d'eau dans la section $B B^{\prime}$ égale ou supérieure d̀ $H_{1}$.

4. Etude du déversement à l'aval du déversoir.

a) Lorsque le niveau de l'eau à l'aval du déversoir est maintenu nettement inférieur à celui de l'eau sur le seuil, la variation du niveau de l'eau dans la région aval du déversoir doit être rapide. Il en résulte que dans cette région, que nous appellerons région $d u$ déversement, une courbure assez prononcée des filets liquides doit se produire; or, il n'est pas tenu compte de semblable courbure dans l'établissement de l'équation différentielle de la surface libre du liquide que nous appellerons surface d'eau. Cette equation s'écrit habituellement sous la forme : 


$$
\frac{d h}{d s}=\frac{i-\frac{\chi}{\omega^{3}} b q^{2}}{\sqrt{1-i^{2}--\frac{q^{2}}{g}} \cdot \frac{l}{\omega^{3}}}
$$

Le terme $-\frac{q^{2}}{g} \frac{. l}{\omega^{3}}$ doit être affecté d'un coefficient $\approx$ supérieur à l'unité, mais dans les applications on le fait généralement égal à $1 . \%$ est le périmètre mouillé.

Cette équation suppose que les sections normales au fond sont hydrostatiques, ce qui n'est suffisamment exact que si la courbure est faible. Elle ne l'est pas dans la région du déversement, et les accélérations normale et tangentielle ont respectivement les sens indiqués par la figure 4, de sorte qu'elles donnent des composantes sur $A B$ dirigées vers le fond. Il en résulte que la projection $j_{t}$ de l'accélération totale sur $\mathrm{AB}$ est positive, et la relation

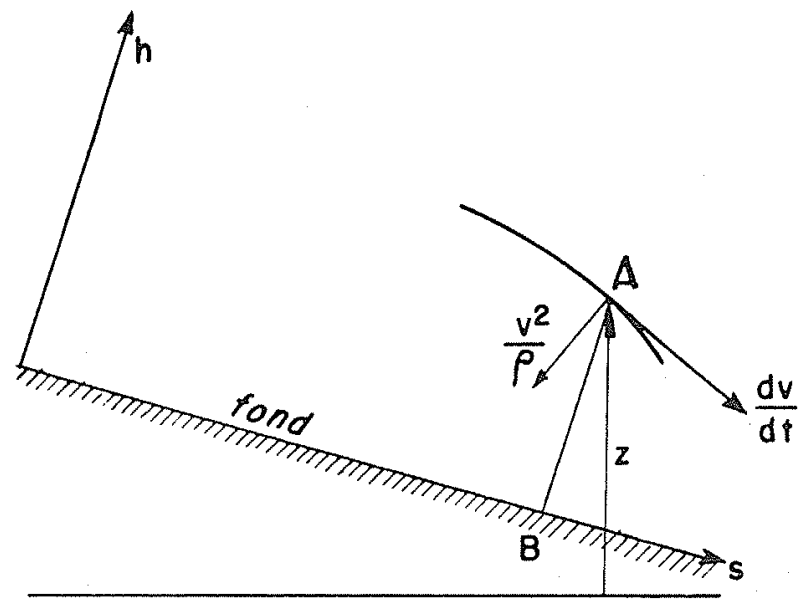

FIG. 4

$$
z_{i}+\frac{p_{\mathrm{R}}}{\gamma}=z_{\lambda}+\frac{p_{\Lambda}}{\gamma}+\frac{1}{g} \int_{i}^{\lambda} \dot{j}_{k} d h
$$

dont le dernier terme est négatif, montre que la pression $p_{\mathrm{R}}$ est inférieure à la pression hydrostatique en ce point.

b) L'équation 5 n'est donc plus applicable dans ce cas, car elle a été déduite de l'équation des quantités de mouvement:

$$
\frac{2}{\Delta}\left[\frac{q U}{g}+\frac{p_{G}}{\gamma}-\frac{\Phi_{s}}{\gamma}\right]=0
$$

basée sur l'hypothèse de la répartition hydrostatique des pressions dans les sections normales an fond.

L'équation 6 doit donc ètre remplacée par une autre dans laquelle le terme $\frac{q \mathcal{U}}{g}$ (que nous po- sons égal à $\zeta_{v}$ ) est remplacé par $\int \frac{v_{\mathrm{s}} d q}{g}$, et le lerme $\frac{\omega p_{a}}{\gamma}$ (que nous posons égal à $\zeta_{p}$ ) est remplacé par $\int \frac{p d \omega}{\gamma} ; v_{s}$ est la projection de la vitesse sur le fond. On obtient ainsi l'équation :

$$
\left.\vec{u}_{1}^{\ddot{1}} \mid \int \frac{v_{s} d q}{g}+\int \frac{p d \omega}{\gamma}-\frac{\Phi_{s}}{\gamma}\right]=0
$$

ou :

$$
\stackrel{\ddot{\Delta}}{i}\left|\varphi_{u}+\varphi_{\nu}-\frac{\Phi_{s}}{\gamma}\right|=0
$$

ou encore :

$$
\stackrel{\ddot{\Delta}}{\stackrel{2}{1}}\left[\vartheta-\frac{\Phi_{s}}{\gamma}\right]=0 \text {, }
$$

en posant :

$$
\int \frac{v_{s} d q}{g}=\varphi_{u} \text { et } \int \frac{p d \omega}{\gamma}=\varphi_{p} \text { et } \varphi_{p}+\varphi_{u}=\varphi \text {. }
$$

Cette équation $7^{\prime}$ est celle de la surface d'eau réelle dans la région du déversement, tandis que l'équation 6 :

$$
\stackrel{\sim}{\Delta}\left|\frac{q \mathrm{U}}{g}+\frac{\omega p_{\mathrm{G}}}{\gamma}-\frac{\Phi_{\mathrm{s}}}{\tau}\right|=0,
$$

ou :

$$
\stackrel{\ddot{\Delta}}{1}\left[\zeta_{n}+\zeta_{p}-\frac{\Phi_{s}}{\gamma}\right]=0
$$

ou encore :

$$
\underset{i}{\ddot{\Delta}}\left\lceil\zeta-\frac{\Phi_{s}}{\gamma}\right\rceil=0
$$

est celle d'une surface théorique pour laquelle l'hypothèse de la répartition hydrostatique des pressions serait réalisée.

Nous répétons que dans les équations 6, les termes en $\frac{q U}{g}$ (U étant la vitesse moyenne) devraient en fait ètre affectés d'un coefficient $\alpha$ supérieur à l'unité. En général, cependant, les lignes d'eau sont calculées avec ces équations 6 ou 5 dans lesquelles a est fait égal à 1 .

c) Ce que nous avons dit de la valeur des pressions réelles dans la zone de déversement montre que :

$$
\varphi_{p}<\zeta_{p} \text {. }
$$

d) D'autre part, toujours dans la région $\mathrm{du}$ déversement, on peut montrer que $\varphi_{u l}>\zeta_{u}$.

$\varphi_{n}$ vaut en effet $\frac{1}{g} \int v_{s}{ }^{2} d \omega$, 
tandis que $\zeta_{u}=\frac{q \mathrm{U}}{g}$ ou $\frac{l}{q} \int \mathrm{U}^{2} d \omega$ avec $\mathrm{U}=\frac{\int v_{\mathrm{s}} d \omega}{\omega}$

Il en résulte que :

$$
\begin{gathered}
\left.\varphi_{u}-\zeta_{u}=\frac{1}{g} \int\left(v_{\mathrm{s}}{ }^{2}-\mathrm{U}^{2}\right) d(\omega)=\frac{1}{g} \iint_{\omega}-\mathrm{U}\right)^{2} d \omega \\
\left.-\frac{2}{g} \int_{\omega} \mathrm{U}^{2} d \omega+\frac{2}{g} \int v_{s} \mathrm{U} d(\omega)=\frac{1}{g} \int_{\omega} v_{s}-\mathrm{U}\right)^{2} d \omega \\
\quad-\frac{2}{g} \zeta_{u}+\frac{2}{g} \mathrm{U}^{2} \omega=\frac{1}{g} \int\left(v_{s}-\mathrm{U}\right)^{2} d \omega
\end{gathered}
$$

puisque $\frac{2}{g} \mathrm{U}^{2}()$ vaut aussi $\frac{2}{g} \zeta_{\eta}$.

Mais si on envisage deux sections $a$ et $b$ de la région du déversement, $b$ étant à l'aval de $a$, on peut montrer que :

$$
\left(\varphi_{u}-\zeta_{u l}\right)_{b}<\left(\hat{\varphi}_{u}-\zeta_{u}\right)_{a} .
$$

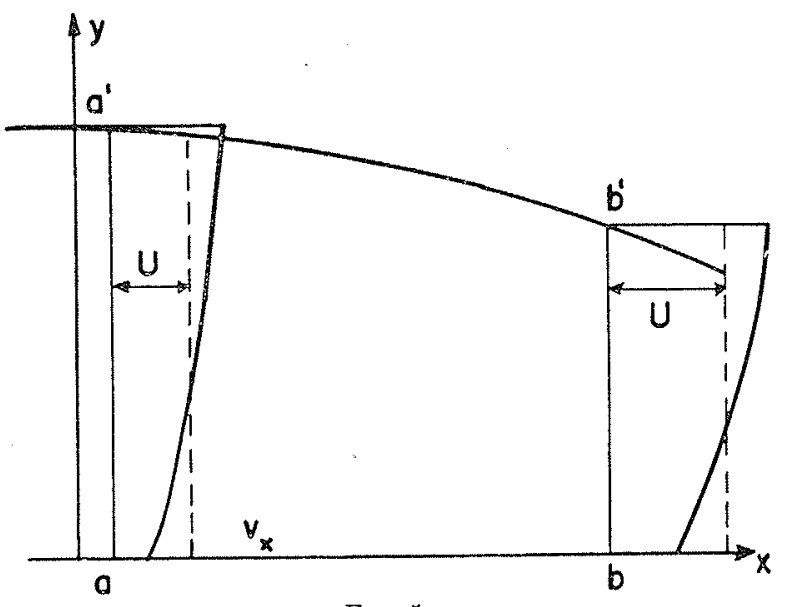

FIG. 5

La tension tangentielle entre deux particules liquide voisines, d'une même section, vaut en effet :

$$
u\left(\frac{\partial v_{x}}{\partial_{x}}+\frac{\partial v_{y}}{\partial_{\|}}\right)
$$

v. étant le coefficient de viscosité.

Supposons d'abord que la variation de vitesse $\Delta v_{i e}$ sur la hauteur $a a^{\prime}$ de la section $a$ soit la même que la variation de vitesse $\Delta v_{x:}$ sur la hauteur $b b^{\prime}$ de la section $b$. Le $\frac{\partial v_{x}}{\partial y}$ moyen de la section $b$ sera alors supérieur au $\frac{\partial v_{x}}{\partial y}$ moyen de la section $a$ : il en est évidemment de même du $\left(\frac{\partial v_{y}}{\partial x}\right)_{b}$ qui est supérieur au $\left(\frac{\partial v_{y}}{\partial x}\right)_{\|}$. I.a tension tangentielle moyenne entre deux particules voisines de $b$ serait par conséquent supé- rieure it la tension tangentielle moyenne entre deux particules voisines de $a$. Or, les tensions tangentielles entre deux particules voisines tendent à uniformiser leurs vitesses en accélérant la plus lente et en ralentissant la plus rapide. Si ces tensions tangentielles entre les particules de $b$ sont supérieures à celles existant en $a$, l'uniformisation des vitesses en $b$ devrait etre mieux réalisée qu'en $a$, ce qui est contraire à notre hypothèse.

L'hypothèse suivant laquelle le $\Delta v_{x}$ sur la hauleur $b b^{\prime}$ est supérieur au $\Delta v$. sur la hauteur $a a^{\prime}$ conduit à un résultat plus absurde encore.

Il en résulte que le $\Delta v_{x}$ entre $b$ et $b^{\prime}$ doit être inférieur au $\Delta v_{x}$ entre $a$ et $a^{\prime}$, c'est-à-dire que les vitesses sont plus uniformément réparties dans la section $b$ que dans la section $a$, ce cui est à rapprocher du résultat analogue pour les conduites convergentes.

On déduit aisément de ce qui précède que :

$$
\left[\frac{1}{g} \int_{\omega}\left(v_{w}-\mathbf{U}\right)^{2} d \omega\right]_{a}<\left[\frac{1}{g} \int_{\omega}\left(v_{w}-\mathbf{U}\right)^{2} d \omega\right]_{a}
$$

En d'autres termes:

$$
\left[\varphi_{v}-\zeta_{v}\right]_{b}<\left[\varphi_{v}-\zeta_{v}\right]_{a}
$$

REMARQUE. - On observera cependant que dans la section de l'extrémité aval du déversoir, pour les nappes déversantes libres, la pression sous la nappe déversante devient égale à la pression atmosphérique. La réduction de pression à laquelle sont soumis les filets inférieurs en passant de l'amont, où la pression est hydrostatique, à l'aval où elle ne vaut plus que la pression atmosphérique, provoque naturellement, dans ces filets, une augmentation de la vitesse qui peut devenir supérieure à celle des couches supérieures. Toutefois, ce phénomène ne se manifeste que sur une très faible étendue, et il disparaît très rapidement vers l'amont comme le montre l'étude de Hun'rer Rouse (4). (Pour un seuil long de $70 \mathrm{~cm}$, avec une nappe déversante d'environ $10 \mathrm{~cm}$ d'épaisseur, la vitesse des couches inférieures est encore la plus faible de toute la section à une distance de $3 \mathrm{~cm}$ de l'extrémité aval du déversoir.)

e) Considérons maintenant l'entièreté de la zone du déversement. Elle est limitée vers l'amont par une région où les faibles courbures rendent d'application l'équation générale 5 .

Soit I la première section, qu'on rencontre en se dirigeant vers l'amont, dans laquelle la loi hydrostatique soit applicable.

Dans cette section, $\varphi_{p}=\zeta_{p}$ ou $\left(\varphi_{p}-\zeta_{p}\right)_{\mathrm{I}}=0$. 
Mais dans la section II, située dans la zone de déversement, $\left(\varphi_{p}-\zeta_{p}\right)_{\mathrm{II}}<0$, de sorte que :

$$
\left(\varphi_{p}--\zeta_{p}\right)_{\mathrm{I}}<\left(\varphi_{p}-\zeta_{p}\right)_{\mathrm{I}} .
$$

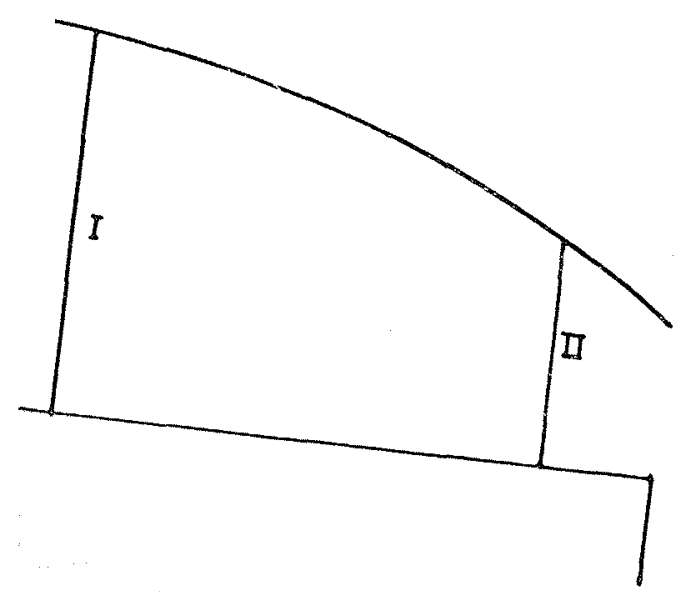

Fic. 6

Datutre part $:\left(\varphi_{u}-\zeta_{u}\right)_{\mathrm{II}}<\left(\varphi_{u}-\zeta_{u}\right)_{I}$.

On en déduit par conséquent que :

$$
{ }_{\mathrm{I}}^{\mathrm{II}}(\varphi-\zeta)<0 \text {. }
$$

et si la section II tend vers $\mathrm{I}$, il vient :

$$
\left[\frac{d(\varphi-\zeta)}{d s}\right]_{r} \leqslant 0
$$

Nous avons signalé plus haut que nous envisagions une surface d'eau réelle satisfaisant à l'équation 7 ou à l'équation suivante qui en est la conséquence :

$$
\left(\frac{d \varphi}{d s}\right)_{r}=\left[\frac{1}{\gamma} \cdot \frac{d \Phi_{s}}{d s}\right]_{,}
$$

Mais nous considérions aussi une surface d'eau théorique répondant à l'équation 6 ou à :

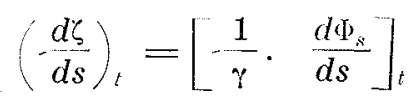

Mais les valeurs des fonctions $\varphi$ et $\zeta$ qui interviennent dans les relations 10 et 11 se rapportent évidemment à la surface réelle qui entrait seule en ligne de compte dans les considérations des paragraphes $c$ et $d$ ci-dessus. La relation 11 peut donc s'écrire :

$$
\left.\left[\left(\frac{d \varphi}{d s}\right)_{r}\right\rceil_{I} \leqslant\left\lceil\frac{d \zeta}{d s}\right)_{r}\right\rceil_{\Gamma}
$$

Dans la section I et en amont de celle-ci, la surface théorique et la surface réelle se confon- dent, de sorte que les valeurs de $\left[\frac{d \Phi_{s}}{d s}\right]$, et de $\left[\frac{d \Phi_{s}}{d s}\right]$, sont les mèmes dans la section I. II en résulte d'après les relations 12 et 13 que :

$$
\left[\left(\frac{d \varphi}{d s}\right)_{r}\right]_{\mathrm{r}}=\left[\left(\frac{d \zeta}{d s}\right)_{t}\right]_{\mathrm{r}}
$$

Les relations 14 et 15 conduisent par conséquent à :

$$
\left[\left(\frac{d \zeta}{d s}\right)_{t}\right]_{\mathrm{r}} \leqslant\left[\left(\frac{d \zeta}{d s}\right)_{1}\right]_{\mathrm{r}}
$$

ou encore :

$$
\left[\left(\frac{d \zeta}{d h}\right)_{1},\left(\frac{d h}{d s}\right)_{,}\right]_{\mathrm{I}} \leqslant\left[\left(\frac{d \zeta}{d h}\right)_{r} \cdot\left(\frac{d h}{d s}\right)_{r}\right]_{I}
$$

et comme :

$$
\left\lceil\left(\frac{d \zeta}{d h}\right)_{r}\right]_{\mathrm{I}}=\left[\left(\frac{d \zeta}{d h}\right)_{t}\right]_{\mathrm{I}}
$$

on peut écrire:

$$
\left(\frac{d h}{d s}\right)_{{ }_{\mathrm{I}}} \leqslant\left(\frac{d h}{d s}\right)_{\mathrm{r}_{\mathrm{I}}}
$$

a condition cependant de ne considérer que des hauteurs d'eau supérieures à $\mathbf{H}_{1}$ pour lesquelles $\frac{d \zeta}{d h}$ est toujours positif.

La dernière relation exprime que pour les hauteur's d'eau supérieures à $H_{1}$, la surface d'eau théorique se trouve en dessous de la surface réelle quand on regarde ver's l'aval.

On pourrait montrer que ces deux surfaces ne peuvent se recouper vers l'aval.

f) Le schéma des surfaces d'eau possibles est donné par les figures 7 ou 8 suivant que la pente de fond est faible ou forte.

Les seules surfaces possibles si la hauteur d'eau est supérieure à $\mathrm{H}_{1}$ sont $\mathrm{A}_{1} \mathrm{~A}_{u}, \mathrm{~A}_{2}$ pour la

$A_{1}$

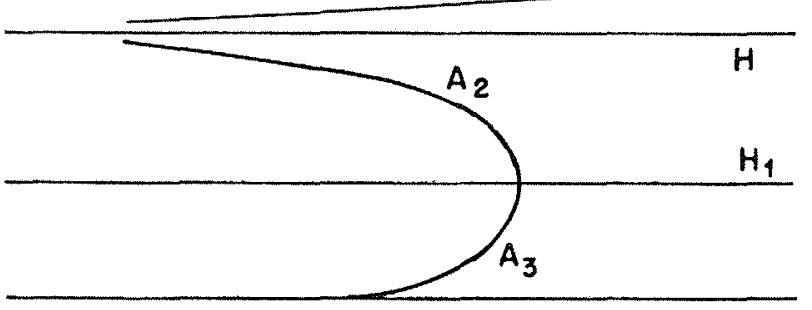

Fig. ? 


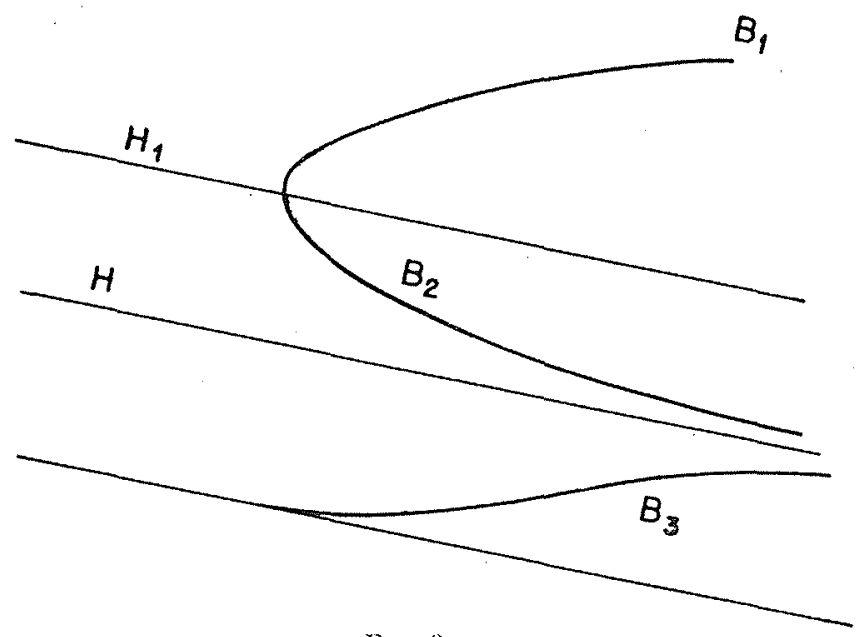

FIG. 8

faible pente de fond et $B_{1}$ pour la forte pente de fond. Ces surfaces d'eau sont celles déduites de l'équation 5 et constituent par conséquent ce que nous avons appelé des surfaces théoriques, distinctes des surfaces réelles dans les seules régions de déversement. Les surfaces théoriques $A_{1}$, $A_{1}$ et $B_{1}$ étant de hauteurs constantes ou croissantes vers l'aval, les surfaces réelles qui y correspondent auront nécessairement des hauteurs croissant vers l'aval, de sorte qu'elles ne pourront se produire en cas de déversement. Seule la surface réelle correspondant da $A_{3}$ pourra se produire dans ce cas.

g) Mais comment déterminer le point de départ de cette surface dans le cas de déversement.

Envisageons d'abord le cas d'une ligne de hauteur $\mathrm{H}_{1}$ coupant la dernicipe section du canal en un point $B$ se trouvant au-dessus de lintersection $\mathrm{C}$ de cette section avec le niveau du réservoir aval. La surface réelle présentera par excmple la forme $R$, et soit 1 la première section vers

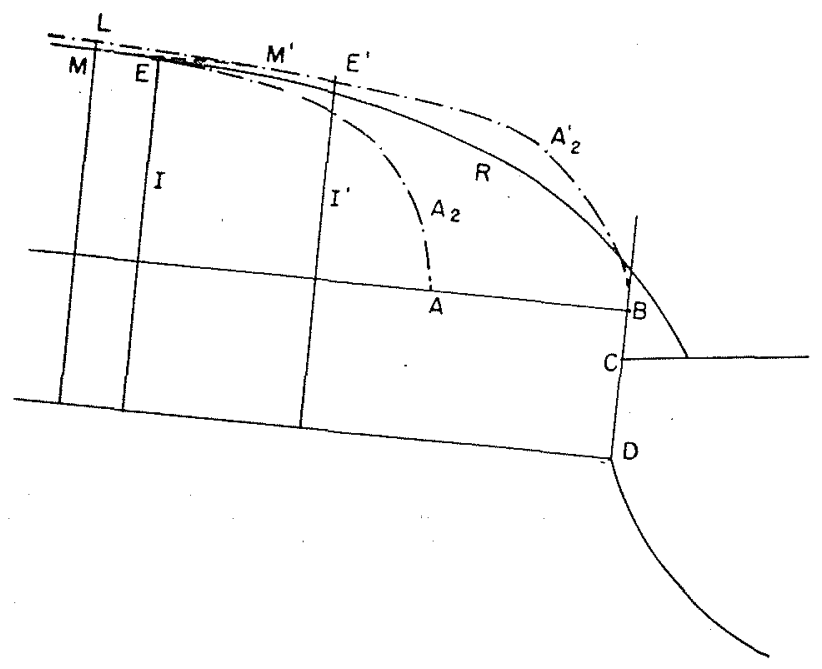

FIG. 9 l'amont dans laquelle les surfaces réelles et théoriques se confondront.

Soit encore $\mathrm{A}_{2}$ la ligne d'eau thérique correspondant à $R$ et se confondant avec cet $R$ en amont de I. Il résulte de la définition même du déversement que la distance de I à la dernière section du canal est faible. Or, la distance entre le point $A$, origine aval de $A_{2}$, et le point $B$ de la dernière section n'est qu'une faible fraction de la Iongueur de déversement.

Faisons maintenant glisser la surface theorique $A_{2}$ parallèlement au fond de facon que le point $A$ vienne en $B$. Ce glissement, avons-nous vu, est de faible amplitude. D'autre part, dans les sections en amont de la section I, les hauteurs de la surface $A_{2}^{\prime}$ provenant du glissement ne diffèrent de celles de la surface théorique et réelle A. que par des différences telles que LM. Or, LM est égal à la différence de hauteurs de sections de la surface $A_{2}{ }^{\prime}$ distantes d'une longueur $M M^{\prime}$ égale à $\mathrm{AB}$.

Mais en amont de I, les courbures étant faibles, la différence des hauteurs dont il vient d'être question, pour un écartement $A B$ que nous avons montré être de peu d'importance, doit nécessairement être très petite. Les différences telles que LM sont donc insignifiantes, et nous remplacerons la surface $A_{2}$ par la surface $A_{2}{ }^{\prime}$. En amont de I, on aura ainsi une surface $\mathrm{A}_{2}^{\prime}$ qui pourra remplacer la surface réelle $\mathrm{R}$; par contre, elle en diffèrera sensiblement dans la région du déversement, ce qui est de peu d'importance vu la faible étendue de cette région.

Cette substitution de $A_{2}^{\prime}$ à $R$ permettra d'avoir un point de départ pour le tracé de la surtace d'eau : ce sera l'intersection $B$ de $H_{1}$ avec la dernière section.

Un exposé analogue montrerait que si ce point d'intersection B se trouve en-dessous du point C. où le niveau du réservoir aval coupe la dernière section, une surface d'eau $A_{2}^{\prime}$ partant de ce point $C$ pourrait pratiquement se substituer à la surface réelle $R$.

5. Etude de l'écoulement sur le seuil d'un déversoir à seuil épais.

a) Il a été établi ci-dessus que l'inllexion à l'amont conduit à une premicre hauteur d'eau, à l'amont, supérieure ou égale à $\frac{a_{\mathrm{m}}}{\sqrt{1-i^{2}}}$

Le diagramme 3 montre que dans ce cas $\mathrm{H}_{1} \ldots h$ (h étant la première hauteur d'eau) est toujours négatif ou nul, c'est-à-dire que la première hauteur $h$ sera supérieure on égale à $\mathrm{H}_{1}$.

b) Si la pente du seuil est faible et notamment si elle est nulle, le schéma de la figure 7 montre que cette première hauteur d'eati à l'amont du déversoir ne pourra être égale à $\mathrm{H}_{1}$ car aucune des surfaces d'eau de cette figure ne commence 
par $H_{1}$. On aura done toujours dans ce cas un débit inférieur au débit maximum avec une des surfaces $A_{2}, A_{i}$ ou $A_{1}$.

a) Si notamment l'eau à l'aval du déversoir se tient en dessous de la hauteur $\mathrm{H}_{1}$ sur le déversoir, il y aura déversement vers l'aval avec production de la surface réelle correspondant à $A_{2}$. Si le problème à résoudre est la détermination du débit $q$ pour une hauteur d'eau $c$ donnée à l'amont du déversoir, il y aura lieu de procéder par tâtonnements. On s"imposera à cet effet nne valeur du débit inférieure au débit maximum. A cette valeur de $q$ correspondra un $\mathrm{H}_{1}$ qui donnera le premier point de la surface d'eau theorique dans la section a (fig. 10). Cette ligne théom rique est alors tracée en utilisant l'équation 5, et on arrive ainsi a une hauteur d'eau $h_{l}$ dans

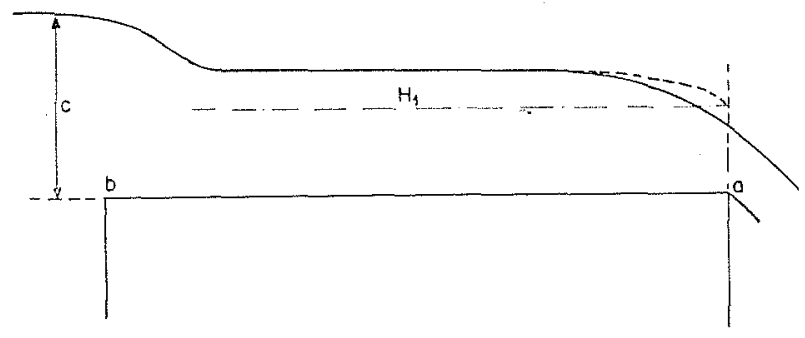

FiG, 10 la section extrême amont du déversoir. On a vu au paragraphe 4 que cette courbe thérique se confond pratiquement avec la ligne réelle à partir d'une section légèrement à l'amont de $a$. D'autre part, la vitesse dans la section $b$ vaut: $v_{b}=\sqrt{ } 2 g\left(c-h_{b}\right), c$ comprenant d'ailleurs la hauteur correspondant à la vilesse d'amence. En multipliant cette vitesse par $\omega_{b}$, correspondant à $h_{b}$ on doit retrouver la valeur du débit utilisée pour la détermination de $\mathrm{H}_{1}$ et le tracé de la surface d'eau. Si, par exemple, la valcur du débil donnée par $\omega_{b}, v_{b}$ est inférieure au débit dont on est parti, il y aura lieu de réduire echii-ci : on peut, en efret, montrer que dans ce cas la ligne d'eau sera abaissée el qu'elle conduira par conséquent à un produit $\omega_{b} . D_{b}$ superieur au premier.

Ces tâtomnements sont toujours asse: longs, et comme en fait on a sourent besoin, pour un déversoir donné, de connaitre les débits correspondants à diverses hauteurs d'eau $c$, il y a avantage à procéder comme suit: on ne part plus d'une hauteur $c$ imposée, mais on se donne un débit qui permet de déduire $\mathrm{H}_{1}$ et de tracer $A_{2}$ partant de eet $\mathrm{H}_{1}$ à l'extrónité aval du barrage, en utilisant l'équation 5 et un coefficient de rugosité pour le seuil. On oblient ainsi $h_{b}$, et par la formule $q=l . h_{b}, 2 g\left(c-h_{b}\right)$, on déduit $c$.

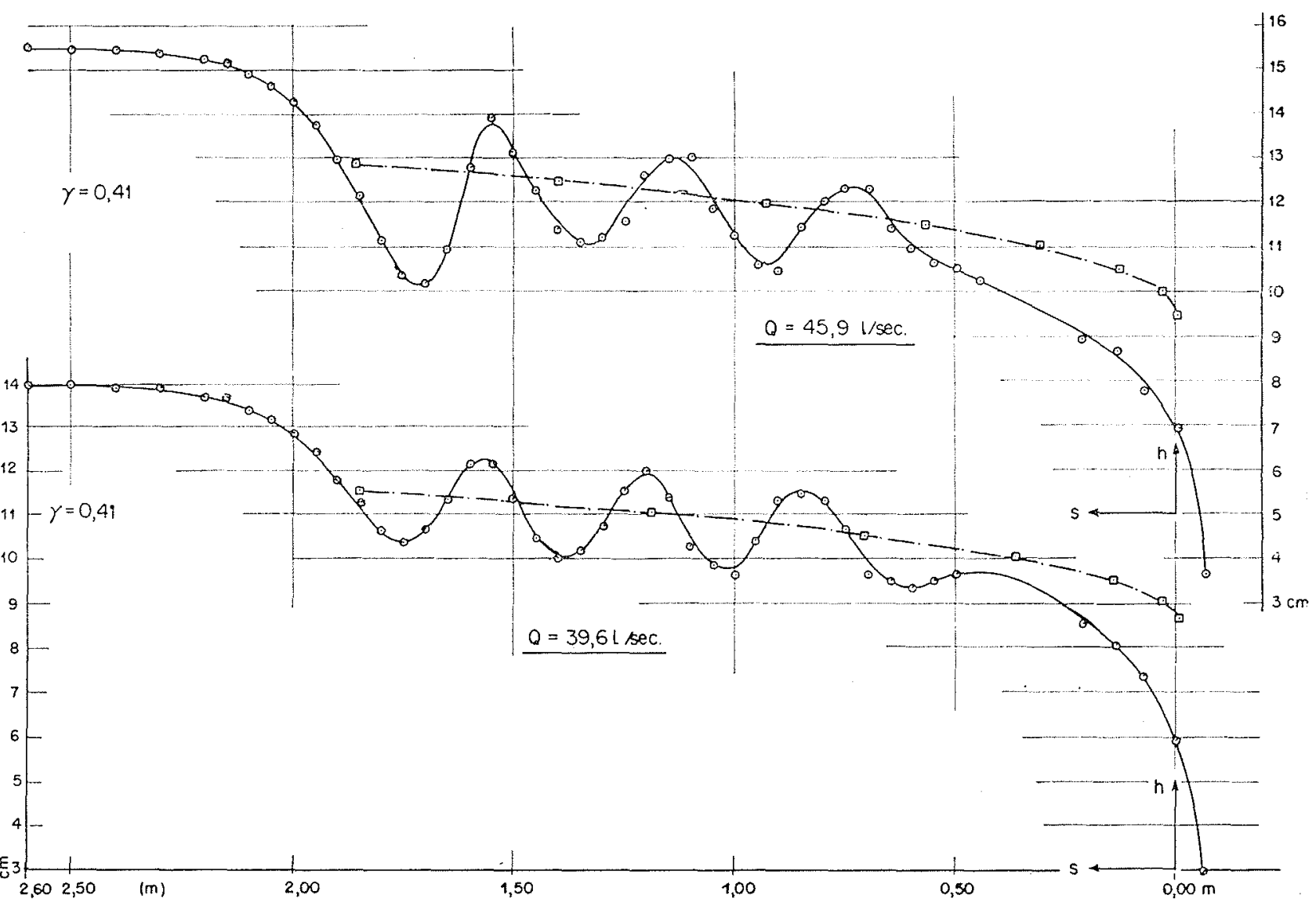

FIG. 11 
Ces opérations répétées avec divers débits permettent de tracer une courbe des débits en fonction de $c$.

Nous avons fait de multiples applications de ce procédé et nous en avons vérifié les résultats par des essais de laboratoire. La concordance est chaque fois excellente. La figure 11 donno pour un déversoir de $0,30 \mathrm{~m}$ de hauteur et de 1,80 $\mathrm{m}$ de longueur, sans contraction à l'amont, le tracé de quelques-unes des lignes d'eau déterminées expérimentalement au laboratoire, et celui des lignes d'eau calculées (résultat final) par le procédé ci-dessus exposé, avec les valeurs des débits mesurées et déterminées par le calcul. On peut se rendre compte de la bonne concordance des résultats. Chacune des figures porte également la valeur du coefficient de rugosité $\gamma$ de la formule de BuzrN du mouvement uniforme :

$$
\mathrm{U}=\frac{-87 \sqrt{\mathrm{Ri}}}{1+\frac{\gamma}{\sqrt{\mathrm{R}}}}
$$

$R$ étant le rayon moyen de la section mouillée.

Certains des essais ont, en effet, eté faits avec une surface du seuil très lisse, tandis que les autres correspondent a un seuil rendu plus rugueux par un cimentage avec sable tris rude.

Le tableau ci-dessous fournit pour les divers essais les valeurs des débits réels, celles des débits calculés par la méthode que nous exposons et, enfin, celles déduites de la formule de BéranGrr. (La largeur du déversoir est de $0,50 \mathrm{~m}$.) $i=o$ et la hauteur $p$ du déversoir sur le fond $=0,30 \mathrm{~m}$.

Tableau I

\begin{tabular}{|c|l|l|l|l|}
\hline $\begin{array}{c}c \\
(\mathrm{~m})\end{array}$ & $\begin{array}{c}q \text { de } \\
\text { BAZIN }\end{array}$ & $\begin{array}{c}q \\
\text { BELANGre } \\
\left(\mathrm{m}^{3} / \mathrm{s}\right)\end{array}$ & $\begin{array}{c}q \\
\text { mesuré } \\
\left(\mathrm{m}^{3} / \mathrm{s}\right)\end{array}$ & $\begin{array}{c}q \\
\text { calculé } \\
\left(\mathrm{m}^{3} / \mathrm{s}\right)\end{array}$ \\
\cline { 1 - 2 } 0,0508 & 0,41 & 0,0098 & 0,00735 & 0,00739 \\
0,0672 & 0,41 & 0,0148 & 0,0115 & 0,0118 \\
0,0875 & 0,41 & 0,0221 & 0,018 & 0,0185 \\
0,104 & 0,41 & 0,02865 & 0,0244 & 0,0242 \\
0,121 & 0,41 & 0,03585 & 0,0308 & 0,031 \\
0,139 & 0,41 & 0,04435 & 0,0396 & 0,0399 \\
0,155 & 0,41 & 0,05075 & 0,0459 & 0,0461 \\
0,081 & 0,13 & 0,01965 & 0,01745 & 0,0179 \\
0,136 & 0,13 & 0,0433 & 0,0407 & 0,0406 \\
\hline
\end{tabular}

On peut cependant se demander si, comme nous l'avons dit ci-dessus, le débit reste inchangé aussi longtemps que le niveau d'aval ne dépasse pas la hauteur $H_{1}$ correspondant à ce débit. Les nappes dont il est question au tableau ci-dessus sont des nappes libres, mais clles peuvent devenir déprimées, plongeantes, ondulées, particulierement lorsque le niveau d'aval est assez élevé. La hauteur $c$ à l'amont du déversoir reste-t-elle effectivement invariable quand la nature de la nappe change, pourvu que le niveau d'aval ne dépasse pas $\boldsymbol{H}_{1}$ ?

Pour vérifier ce point nous avons observé l'écoulement de divers débits avec nappe libre, avec nappe déprimée, avec nappe adhérente, et en faisant monter le niveau d'aval, avec nappe plongeante et nappe ondulée. Le tableau cidessous donne le résultat des essais pour l'un de ces débits : il montre que la hauteur $c$ à l'amont du déversoir ne varie pas d'une facon observable aussi longtemps que le niveau daval ne dépasse pas la hauteur $H_{1}$. Ce fait est d'ailleurs compréhensible à l'aide de ce qui a été exposé sous le chiffre 4. La répartition des pressions dans les sections normales au fond est évidemment modifie par la forme de la nappe, mais cette modification est pratiquement limitée anx dernières sections d'aval du seuil. Le changement de la nature de la nappe ne pourrait avoir une influence sur la valeur de c que si la longueur du déversoir était insuffisante pour que la nappe s'étende vers l'amont en dehors de la région du déversement dans laquelle nous savons que la surface A: n'est pas substiluable à la surface réelle. D'après nos essais, même avee une valeur de $c$ égale à $0,16 \mathrm{~m}$ et une hautcur $p$ de déversoir de $0,30 \mathrm{~m}$, cette longueur du déversoir ne deviendrait insuffisante que si elle tombait en dessous de $0,60 \mathrm{~m}$. Dans ce cas, les développements qui précèdent seraient évidemment inutilisables.

\section{Tableau II}

$q=0,0124 \mathrm{~m}^{3} / \mathrm{s}$ pour une largeur de $0,50 \mathrm{~m}$.

Longueur du déversoir : $1,80 \mathrm{~m} .-i=0$.

\begin{tabular}{|c|c|c|c|c|}
\hline $\begin{array}{l}\text { Nature de } \\
\text { la nappe }\end{array}$ & 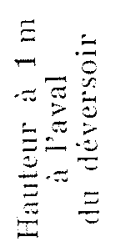 & 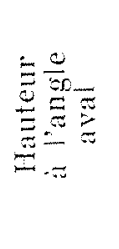 & 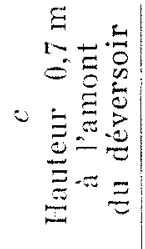 & $\Xi$ \\
\hline $\begin{array}{l}\text { libre..... } \\
\text { ondulèe... } \\
\text { plongeante } \\
\text { ondulée... } \\
\text { ondulée. . } \\
\text { ondulée... } \\
\text { ondulée... } \\
\text { ondulée. . }\end{array}$ & $\begin{array}{c}" \\
n \\
0,0178 \\
0,0078 \\
0,0211 \\
0,0375 \\
0,0449 \\
0,0524\end{array}$ & $\begin{array}{l}0,0238 \\
0,0225 \\
0,023 \\
0,0245 \\
0,03 \\
0,0375 \\
0,0455 \\
0,0518\end{array}$ & $\begin{array}{l}0,0683 \\
0,0683 \\
0,0683 \\
0,0683 \\
0,0683 \\
0,0683 \\
0,0686 \\
0,07\end{array}$ & $\begin{array}{l}0,0395 \\
0,0395 \\
0,0395 \\
0,0395 \\
0,0395 \\
0,0395 \\
0,0395 \\
0,0395\end{array}$ \\
\hline
\end{tabular}


Nous ferons mème, dans la suite, une remarque suivant laquelle la hauteur à l'aval du déversoir peut être légèrement supérieure à $\dot{H}_{1}$ sans que la valeur de c pour la nappe libre soit modifiée.

ß) Si l'eau dans le canal a l'aval du déversoir se tient à une hauteur comprise entre les hauteurs $\mathrm{H}_{1}$ et $\mathbf{H}$ (hauteur du mouvement uniforme) sur le déversoir, il se produit encore une surface d'eau $\mathrm{A}_{2}$ dont le premier point vers l'aval est, cette fois, le point d'intersection du niveau de l'eau dans le canal à l'aval du deversoir avec la dernière section d'aval du déversoir. Le calcul du débit se fera encore en determinant la surface A. mais en prenant le point de départ qui vient d'être indiqué. Ce calcul exige, par conséquent, non seulement la connaissance de $c$ comme précédemment, mais aussi celle de $h_{u}$ (fig. 12).

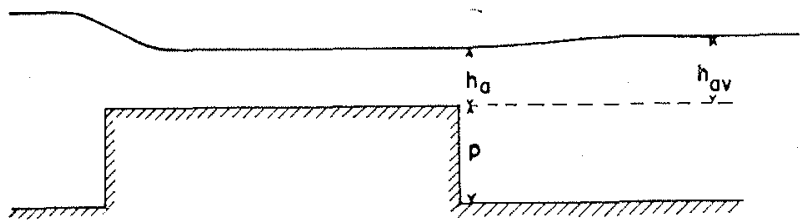

Fig. 12

Il y a, toutefois, lieu de remarquer que $h_{\mathrm{i}}$ ne coïncide avec la hauteur $h_{a n}$ dans le canal à l'aval du déversoir que si la vitesse dans ce canal est très faible.

Comme l'a montré Escandr: $h_{a n}$ est en fait supérieur à $h_{a}$. En appliquant l'équation de Bernoulla entre les sections $a$ et $a v$, on obtient:

$$
\left\lceil h_{c}=h_{a v}-\frac{\mathrm{U}_{a v}}{g}\left(\mathrm{U}_{a}-U_{a v}\right)\right]
$$

en tenant compte de la perte de charge à la sortie de la section $a$.

Cette équation permet de trouver $h_{\text {" }}$ pour un

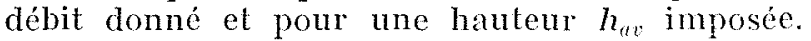
Partant de la hauteur $h_{\text {u }}$ ainsi obtenue, on pourra, avec le débit donné et la rugosité du seuil, tracer la surface $A_{2}$ : d'où $h_{b}$ et, par conséquent, $c$ à l'aide de la formule :

$$
q=l . h_{b} \vee 2 g\left(c-h_{b}\right) .
$$

Il sera dès lors possible de tracer un diagramme $\dot{a}$ deux entrées $c$ et $h_{a r}$ avec courbes de débit constant. C'est à celte remarque que nous faisions allusion au paragraphe $\%$ ci-dessus.

y). Si le niveau de l'eau à l'aval du déversoir est à une hauteur $H$ du mouvement uniforme, on aura la surface $A_{w}$, et si la hauteur en question est supérieure à $H$, on aura $A_{1}$. Le calcul du débit dans ce cas, ou mieux la détermination de l'abaque à deux entrées donnant $q$ en fonc- tion de $h_{a r}$ et de c se fait d'ailleurs comme il est indiqué au paragraphe $\beta$.

c) Si la pente de fond est forte sur le scuil, la figure 8 montre que la première hauteur d'eau à l'anlont peut dans ce cas être égale à $\mathrm{H}_{1}$, pour donner naissance à l'une des surfaces $B_{1}$ ou $B_{2}$.

a) Si leau du canal à l'aval du déversoir se tient à un niveau inférieur à $\mathrm{H}_{1}$, hautcur critique correspondant au débit maximum, on aura une surface d'eau $B_{\text {. }}$ avec un débit égal au débit maximum. La formule de Bécaxger est, dans ce cas, applicable (pour les sections rectangulaires).

ß) Il en est de même si l'eau du canal à l'avai du déversoir se tient à une hauteur telle qu'un ressaut se produise soit à l'aval du déversoir; soit sur le déversoir. Le débit sera encore le débit maximum. On peut se rendre compte s'il en sera ainsi par calcul, en tracant la surface d'eau $B_{2}$ avec le débit maximum et en partant de la hautcur $\mathrm{H}_{1}{ }^{\prime}$ (hauteur critique correspondant au débit maximum) dans la section $b$ (fig. 13). Si $h_{a}$,

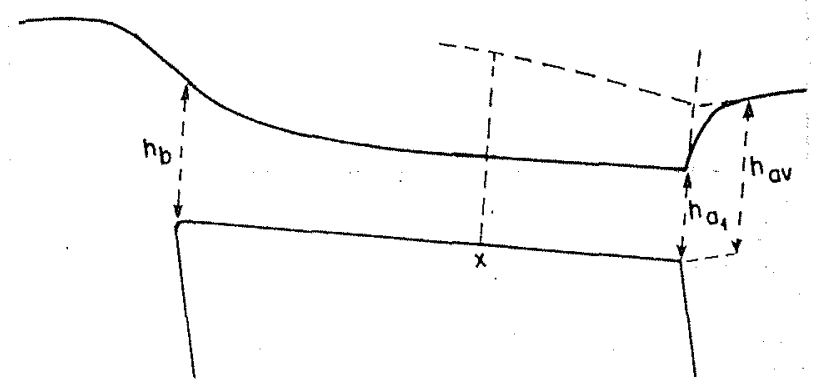

FIG. 13

hauteur à l'aval du déversoir, est inférieure ou égale à la hauteur $h_{a 2}$ qui correspond après ressaut à la hauteur $h_{a 1}$ avant ressaut qui termine la surface $B_{2}$ vers l'aval, le ressaut se fera dans la section a ou à l'aval de celle-ci. La détermination de $h_{a j}$ en partant de $h_{a^{1}}$ se fait en utilisant l'équation :

$$
\left({ }_{(1)} \frac{p_{:}}{\gamma}+\frac{q \mathrm{U}}{g}\right)_{a_{3}}=\left(\frac{\omega p_{\mathrm{r}}}{\gamma}+\frac{q \mathrm{U}}{g}\right)_{a_{1}} .
$$

Si $h_{a r}$ esl supérieure à $h_{a 2}$, on recherchera par la méthode habituelle de la courbe des ressauts si une surface $B_{1}$ commencant dans la section $a$ avec la hauteur $h_{a t}$ et tracée avec le débit maximum ne donnera pas un ressaul avec la surface $B_{.}$du débit maximum dans une section $x$ du déversoir. Dans l'affirmative, le débit ace le debil maximum et la formule de BécasGER reste toujours applicable pour les lits rectangulaires.

$\gamma)$ Si le ressaut est impossible quand $h_{r 1}$ est supérieur à $H_{1}, B_{1}$ doit exister sur toute la lon- 
gueur, et son tracé, comme la détermination du débit, exige la connaissance non seulement de $c$ mais aussi de $h_{a v}$. Le probleme devient alors le même qu'au paragraphe b) ß) ci-dessus.

On peut encore se demander si les considérations theoriques des paragraphes relatifs à la forte pente du seuil se vérifient dans les essais et si notamment les fortes courbures de l'inflexion ne provoquent pas des modifications des résultats présentés.

Nous avons donné au seuil du déversoir ayant servi aux essais précédemmont décrits une pente de 0,0124. Le tableau ci-dessous montre que lorsque $h_{t i v}$ est inférieur à $H_{1}$, la formule de BéLANGTr est bien applicable, comme il résulte des considérations que nous avons émises.

Tableav III

\begin{tabular}{|c|c|c|c|}
\hline $\begin{array}{c}c \\
(\mathrm{~m})\end{array}$ & $\begin{array}{c}q \\
\text { (formule } \\
\text { BÉLANGER) } \\
\left(\mathrm{m}^{3} / \mathrm{s}\right)\end{array}$ & $\begin{array}{c}q \\
\text { (mesuré) } \\
\left(\mathrm{m}^{3} / \mathrm{s}\right)\end{array}$ & Observations \\
\hline $\begin{array}{l}0,045 \\
0,0617 \\
0,112\end{array}$ & $\begin{array}{l}0,0081 \\
0,0131 \\
0,0319\end{array}$ & $\begin{array}{l}0,008 \\
0,0132 \\
0,032\end{array}$ & $\begin{array}{l}\text { Largeur : } \\
0,50 \mathrm{~m}\end{array}$ \\
\hline
\end{tabular}

Les résultats ci-dessus sont cependant en opposition avec ceux de Woonburs qui, pour les déversoirs à forte pente, avec ou sans ressaut, mais avec nappe $B_{2}$, trouve des débits réeìs parfois notablement inférieurs à ceux donnés par la formule de Bŕanger. Selon cet auteur, pour ces déversoirs inclinés, le coefficient de débit décroît quand la charge augmente jusqu'à ce qu'elle atteigne 1,3 pieds. Ce coefficient reste alors constant pour des charges plus fortes.

Nous estimons que les résultats de Woonburn sont provoqués par le rayon de courbure insuftisant du raccordement du seuil du déversoir avec sa paroi verticale d'amont ( $R: 6$ pouces). Pour les faibles charges et les petites vitesses, la contraction inférieure est faible et les résultats concordent pratiquement avec ceux de la formule de BÉLavger. Mais la contraction augmente avec la charge pour tendre cependant vers une constante aux très grandes valeurs du nombre de Reynolds (comme dans les orifices) : le débit réel devient alors inférieur à celui déduit de la formule de Bridanger.

Nous croyons d'ailleurs trouver la preuve qu'il en est bien ainsi dans une intervention de M. Parshall dans la discussion qui a fait suite à la publication de Woonburx. Parshali a travaillé avec divers déversoirs à seuil épais de forle pente en s'efforcant de réaliser un raccordement continu à grand rayon de courbure vers l'amont.
Tous ses résultats satisfont à la formule de BÉLANGER pour les essais des types $c$ ) $\alpha$ ) et $\beta$ ) cidessus.

REMARQUE. - Il faut observer que la forte ou la faible pente n'est pas uniquement détermince par la valeur de la pente. C'est ainsi que dans certains de nos essais avec la pente de fond de 0,0124, la pente est faible et la surface de la nappe est du type A.z (par exemple pour un débit de $0,0056 \mathrm{~m}^{3} / \mathrm{s}$, sur une largeur de $0,50 \mathrm{~m}$ ). Pour d'autres essais, avec la mème pente de fond, la pente est forte et la surface est du type $B_{2}$ (par ex. pour un débit de $0,032 \mathrm{~m}^{3} / \mathrm{s}$, sur une largeur de $0,50 \mathrm{~m}$ ).

Il faut, par conséquent, contrôler la nature de la pente de fond dans les calculs dont les principes ont élé exposés ci-dessus, chaque fois que le doute est possible, par exemple en comparant les valeurs de $\mathrm{H}$ et de $\mathrm{H}_{1}$.

6. Les ondulations de la surface. - La théorie de ces ondulations a été faite par Boussrneso (5). $\mathrm{Si}$ on considère une surface libre d'un cours d'eau de hauteur $\mathrm{H}+\eta=h$, voisine de la hauteur du mouvement uniforme $H$, Boussineso trouve pour $\eta_{1}$ l'expression :

$$
\eta=\mathrm{A} e^{r_{1} x}+\mathrm{B} e^{-1 / 2 r_{1} x} \sin y(x-\mathrm{C}) \text {; }
$$

$A, B$ et $C$ sont des constantes d'intégration, $i_{1}$ est la racine positive de l'équation :

$$
r^{3}-\frac{3}{\mathrm{H}^{2}}\left(1-\frac{g \cdot b}{i}\right) r-9 \frac{f \cdot g \cdot b}{\mathrm{H}^{3}}=0,
$$

$b$ étant le coefficient de rugosité correspondant à $\mathrm{H}$, et $f$ est un coefficient valant sensiblement 1,1. Quant à v, il est donné par :

$$
y^{2}=\frac{9 f \cdot g \cdot b}{H^{3} \cdot r_{1}}-\frac{r_{1}^{2}}{2},
$$

si :

$$
i<\frac{g \cdot b}{1-\frac{1}{4}[36 f \cdot g \cdot b]^{2 / 3}}
$$

Si on étudie la région qui précède celle où le mouvement uniforme est établi, $r_{i}$ doit s'annuler pour $x$ positif et tres grand (moyennant un choix convenable de l'origine) et A doit être extrêmement petit. L'expression de $\eta$ se réduit alors à son second terme:

$$
\eta=\frac{\mathrm{B}}{e \frac{r_{1}}{2} x} \sin \nu(x-\mathrm{C}) \text {. }
$$


Dans lapplication aux ondulations se produisant sur le déversoir à seuil épais, nous assimilerons à $\mathrm{H}$, hauteur du mouvement uniforme, la hauteur autour de laquelle la nappe oscille dans la partie amont et centrale du déversoir de facon à éliminer la partie de la nappe influencée par le déversement. A cette hauteur $H$ correspond une pente $i$ qui permet de calculer $r_{1}$ et par suite $\nu$; la longueur d'onde des ondulations complètes est alors donnée par $2 \pi / \%$.

Nous avons d'ailleurs été conduit à une autre expression de cette longueur d'onde par les considérations suivantes.

La vitesse dans la section 1 (fig. 14) est

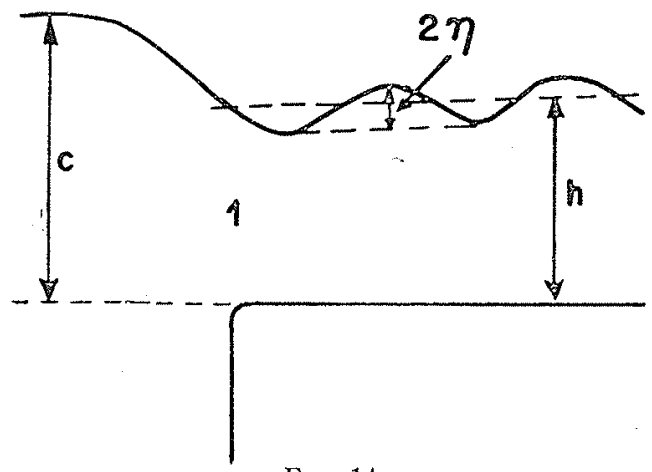

Fig. 14

connue par les calculs qui précèdent, $q$ et $h$ ayant été déterminés. Ces calculs supposent que la section 1 est hydrostatique. Mais la production des courbures et les variations de hauteur dues aux oscillations peuvent modifier la répartition des pressions. Toutefois, comme le débit réel a très sensiblement la valeur du débit calculé, c'est que les deux influences qui viennent d'être citées s'équilibrent. Ainsi, dans la section 1 , la réduction de pression due à la diminution de la hauteur doit être compensée par une augmentation de pression due à la courbure vers le haut des trajectoires. Au sommet inférieur de la première ondulation, la courbure $\frac{1}{0}$ vaut $\frac{d x^{2}}{d^{2} y}$, soit $r_{11} \nu^{2}$, l'équation des ondulations étant $y=r_{1} \sin \vee x$.

Mais la courbure doit varier de la surface au fond où elle est nulle : admettons que cette variation soit linéaire. D'autre part, l'augmentation de pression au fond, résultant de ces courbures, vaut $\frac{\gamma}{g} \int_{0}^{h} \frac{v^{2}}{\rho} d h, \gamma$ étant le poids spécifique de l'eau. En tenant compte de la valeur de o à la surface et de la répartition admise pour p, l'augmentation de pression prend l'expression :

$$
\frac{\gamma}{g} \cdot \frac{v^{2} \cdot \eta_{11} \cdot \nu^{2}}{2} \cdot h
$$

Par contre, la dénivellation à l'endroit considéré amènera au fond une réduction de pression égale à $\% \eta_{11}$, et, du fail de la compensation des deux actions, on a :

$$
h \cdot \frac{b^{2} \cdot \eta_{1} \cdot v^{2}}{2 \cdot g}=r_{11} \quad \text { oll } \quad v^{2}=\frac{2 \cdot g}{v^{2} \cdot h}
$$

la longueur d'onde d'une osciliation totale vaut done :

$$
v \cdot \pi \cdot \sqrt{\frac{2 h}{g}}
$$

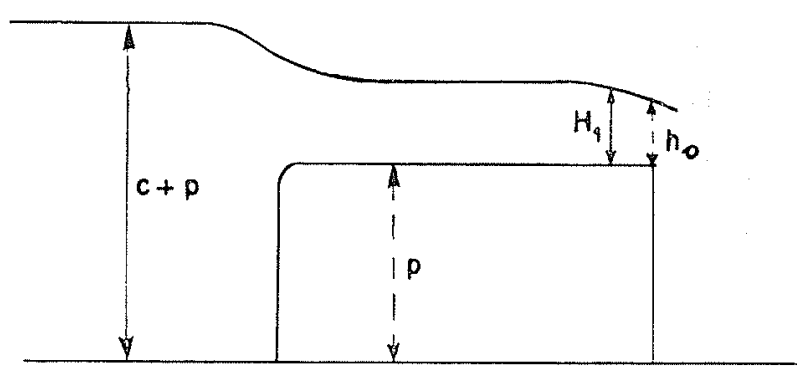

FIG. 15

L'amplitude de ces oscillations est fonction de la raideur de l'inflexion. Plus celle-ci est brusquement descendante et plus l'amplitude des oscillations est grande. Or, la raideur de l'inflexion ou chute de l'entrée est influencée par la hauteur du déversoir et par la façon dont il est raccordé au fond vers l'amont. Les fables hauteur's de déversoir et les raccordements doux et continus donnent des chutes donces et, par suite, de faibles amplitudes des oscillations.

Le tableau suivant donne, pour diverses nappes relevées en notre laboratoire, les valeurs de $r_{1}$, de $\nu$, celles de la longueur d'onde $\lambda$ calculée d'après l'expression de Boussineso, calculée ensuite par le procédé que nous avons exposé, ainsi

\begin{tabular}{|c|c|c|c|c|c|}
\hline \multirow[b]{2}{*}{$q$} & \multirow[b]{2}{*}{$r_{1}$} & \multicolumn{3}{|c|}{$\lambda=$ longucur d'onde } & \multirow[b]{2}{*}{$\begin{array}{c}\text { Chute } \\
h^{\prime}\end{array}$} \\
\hline & & 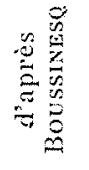 & 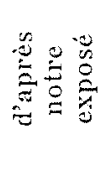 & 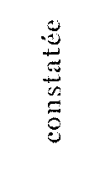 & \\
\hline 0,0072 & 64 & 0,098 & 0,106 & 0,105 & 0,0065 \\
\hline 0,0115 & 42 & 0,14 & 0,14 & 0,135 & 0,012 \\
\hline 0,018 & 32,6 & 0,192 & 0,20 & 0,20 & 0,014 \\
\hline 0,0244 & 26 & & 0,24 & 0,24 & 0,018 \\
\hline 0,0308 & 21,5 & & 0,295 & 0,30 & 0,022 \\
\hline 0,0396 & 17 & & 0,35 & 0,365 & 0,027 \\
\hline 0,0459 & 15 & & 0,41 & 0,41 & 0,035 \\
\hline
\end{tabular}
que la longueur d'onde mesurée.

\section{TABleau IV}


7. Calcul rapide du débit dans le cas d'une faible pente du seuil $i$, avec déversement vers l'aval en nappe libre.

Hunter Rouse (Fluid Mechanice for hydraulic Engineers, page 324) indique que dans ce cas la hauteur $h_{0}$ sur la crête aval du déversoir vaut
$0,715 \mathbf{H}_{1}$, et comme $q^{2}$ vaut $g \cdot \mathrm{H}_{1}^{3}$, la mesure de $h_{0}$ permettrait la détermination rapide du débit (lit rectangulaire).

Le tableau suivant donne les résultats de nos mesures pour diverses nappes libres ave faible pente du fond du seuil ( $p$, la hauteur du déversoir, est égale à $0,30 \mathrm{~m}$ ).

Tableav V

\begin{tabular}{|c|c|c|c|c|c|c|c|c|}
\hline$q$ & $i$ & de $\stackrel{\gamma}{\gamma}$ AZIN & $c$. & $\mathrm{H}_{1}$ & $h_{0}$ & $\frac{h_{0}}{\mathrm{H}_{1}}$ & $\frac{c}{\mathrm{H}_{1}}$ & $\frac{p}{c}$ \\
\hline 0,0072 & 0 & 0,4 & 0,0508 & 0,028 & 0,0215 & 0,77 & 1,82 & 6 \\
\hline 0,0115 & 0 & 0,4 & 0,0672 & 0,0378 & 0,0285 & 0,753 & 1,77 & 4,5 \\
\hline 0,018 & 0 & 0,4 & 0,0875 & 0,0508 & 0,038 & 0,748 & 1,724 & 3,425 \\
\hline 0,0224 & 0 & 0,4 & 0,104 & 0,0624 & 0,0445 & 0,713 & 1,665 & 3 \\
\hline 0,0308 & 0 & 0,4 & 0,121 & 0,073 & 0,0525 & 0,718 & 1,655 & 2,5 \\
\hline 0,0396 & 0 & 0,4 & 0,139 & 0,0862 & 0,06 & 0,69 & 1,614 & 2,16 \\
\hline 0,0459 & 0 & 0,4 & 0,155 & 0,095 & 0,069 & 0,725 & 1,63 & 2 \\
\hline 0,00641 & 0 & 0,13 & 0,044 & 0,0254 & 0,0185 & 0,73 & 1,735 & 6,8 \\
\hline 0,01745 & 0 & 0,13 & 0,0825 & 0,0496 & 0,0375 & 0,756 & 1,66 & 3,65 \\
\hline 0,02915 & 0 & 0,13 & 0,116 & 0,0697 & 0,0525 & 0,753 & 1,665 & 2,58 \\
\hline 0,055 & 0 & 0,13 & 0,165 & 0,1065 & 0,075 & 0,705 & 1,55 & 1,82 \\
\hline 0,0056 & 0,0124 & 0,40 & & 0,0232 & 0,0135 & 0,58 & & \\
\hline
\end{tabular}

Ce tableau montre que, dans nos essnis, le rapport $\frac{h_{0}}{H_{1}}$ est très variable.

Quand on traite le problème théoriquement, on ne voit d'ailleurs pas très bien la raison pour laquelle ce rapport doit être constant. Ainsi, si on applique le théorème des quantités de mouvement entre une section à l'amont du déversoir où la hauteur totale est $p+c$, et la section du déversoir dans laquelle $h=\mathrm{H}_{1}$, on obtient, en tenant compte de la réaction horizontale de la paroi amont du déversoir mais sans faire intervenir les réactions tangentielles du seuil :

$$
\frac{c^{2}}{2}+\frac{q^{2}}{g \cdot(p+c)}=\frac{\mathrm{H}_{1}^{2}}{2}+\frac{q^{2}}{g \mathrm{H}_{1}},
$$

et comme $q^{2}$ vaut $g \mathrm{H}_{1}{ }^{3}$, cette relation devient :

$$
\frac{c^{2}}{2}+\frac{\mathrm{H}_{1}^{3}}{p+c}=\frac{3 \cdot \mathrm{H}_{1}^{3}}{2}
$$

Le rapport $\frac{c}{\mathrm{H}_{1}}$ est done une fonction de $p$ que la résolution par tâtonnements de l'équation 17 permet de mettre sous la forme du diagramme 16.

Mais cette relation est malheureusement modifiće quand on tient compte des réactions tangen- tielles du seuil. Mais, dans ce cas, le problème ne peut plus être traité d'une facon générale, car ces réactions dépendent des hauteurs d'eau, de la rugosité, de la longueur du seuil, etc.

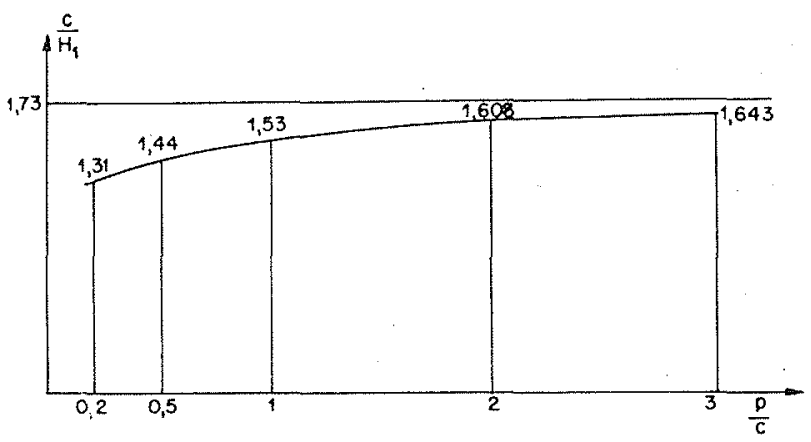

FIG. 16

Nous avons résolu l'équation 17 complétée par ce terme des réactions tangentielles du seuil, pour notre déversoir, dans quelques cas particuliers. $(\gamma=0,4$ et $\mathrm{L}=1,80 \mathrm{~m}$. $)$

On trouve :

pour

$\begin{array}{cc}P & c \\ c & \mathrm{H}_{1} \\ \infty & 1,84 \\ 3 & 1,70 \\ 2 & 1,64 .\end{array}$


Pour obtenir la valeur du rapport $\frac{h_{0}}{H_{1}}$, nous pouvons procéder de la même manière et appliquer le théorème des quantités de mouvement entre la section de hauteur $\mathrm{H}_{1}$ et la section terminale de hauteur $h_{0}$. Sans tenir compte des réactions tangentielles du lit, c'est-à-dire du seuil, entre ces deux sections et en adoptant pour la répartition des pressions dans la section $h_{0}$ celle indiquée par Hunter Rouse (Verteilung der hydraulischen Energie bei einen lotrechten Absturz), cette équation s'écrit :

$$
\frac{\mathrm{H}_{1}^{2}}{2}+\frac{q^{2}}{g \cdot \mathrm{H}_{1}}=0,1715 h_{0}{ }^{2}+\frac{q^{2}}{g h_{0}}
$$

et en observant que $q^{2}=g \cdot H_{1}{ }^{3}$, on oblient :

$$
1,5 \frac{h_{61}}{\mathrm{H}_{1}}=0,1715 \frac{h_{0}{ }^{3}}{\mathrm{H}_{1}{ }^{3}}+1
$$

relation qui conduit à la valeur 0,712 pour le rapport $\frac{h_{1}}{h_{1}}$, valeur très concordante avec celle de Hunter Rouse.

'Toutefois, il n'a pas été tenu compte des réactions tangentielles du seuil entre ces deux sections. Ces réactions vont donner un terme positif dans le second membre des relations cqui précèdent, ce qui va augmenter la valeur de $\frac{h_{0}}{\mathrm{~F}_{1}}$, d'autant plus que ces réactions seront relativement grandes. Le terme à ajouter au second nembre de l'égalité 18 est égal à la perte de charge entre les deux sections mutipliée par la section et $h_{0}$ et divisée par $\mathrm{H}_{1}^{*}$, c'est-a-dire:

$$
\frac{\Perp}{()^{2}} \cdot b \cdot q^{2} \cdot \frac{h_{01}}{\mathrm{H}_{1}^{*}} \mathrm{~L}^{\prime}
$$

$L^{\prime}$ étant la distance entre les deux sections considérées.

Nous avons calculé ce terme pour certains des essais dont les résultats ont été mentionnés cidessus et sont notamment partielemenl repris par les figures 11 et 11 bis. Ainsi, pour le débit de $0,0459 \mathrm{~m}^{3} / \mathrm{s}$, il ne faul ajouter au second membre de l'équation 18 que 0,0016 , ce qui donne pour la solution exactement : 0,715. Par contre, pour le débit de $0,0115 \mathrm{~m}^{3} / \mathrm{s}$, le terme à ajouter devient 0,04 , et la solution est portée à 0,74 . Ajoutons cependant que ces calculs du terme complémentaire ne sont qu'approximatifs.

Ils ont cependant l'avantage de montrer que, conformément aux résultats des essais, le rapport $\frac{h_{0}}{H_{1}}$ augmente pour les petits débits. Disons cependant que la valeur 0,715 semble pouvoir être utilisée pour lés déversoirs à seuil lisse, relativement courts et pour des débits relativement élevés.

\section{BHBLOGRAPHE}

(1) BAZIN. - Recherches sur les déversoirs. (Annales des Ponts et Chanssées, 1895 in 1898.)

(2) ESCANDE. - Le principe du maximum du débit des déversoirs à seuil épais.

(Génie Civil, 1942.)

(3) HUNTER ROUSE. - Fluid Mechanics for hydraulic Engineers.

HUNTER ROUSE. - Verteilung des Hydraulischen Energie bei einem lotrechten Absturz. Oldenbours, 1933.

(t) J. G. WOODBURN. - Tests of Broad-crested Feirs. Procedings American Society of Civil Engineers, 1930. La diseussion dans les numéros suivants.

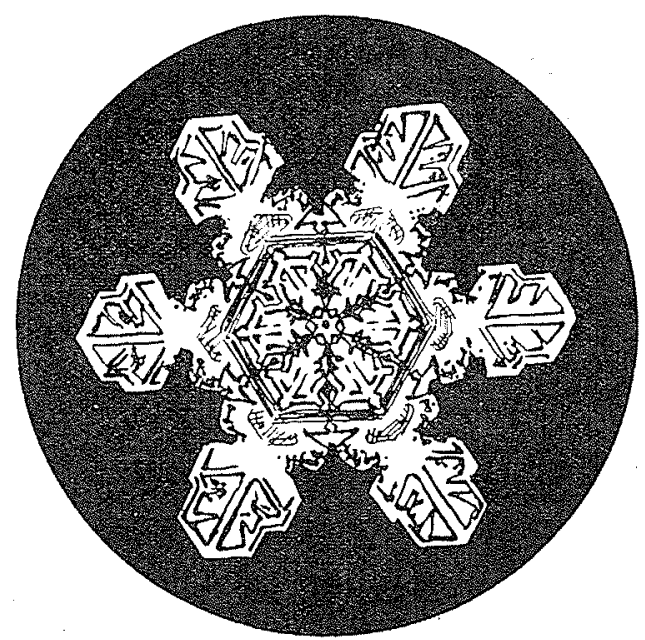

